\title{
Intelligent image analysis for retrieval of leaf chlorophyll content of rice from digital images of smartphone under natural light
}

\author{
P. JAGAN MOHAN and S. DUTTA GUPTA ${ }^{+}$ \\ Agricultural and Food Engineering Department, Indian Institute of Technology Kharagpur, \\ Kharagpur 721302, India
}

\begin{abstract}
The present study describes a new imaging method to acquire rice leaf images under field conditions using a smartphone and modeling approaches to retrieve the leaf chlorophyll $(\mathrm{Chl})$ content from digitized images. Pearson's correlation of image-based color indices of the relative Chl content measured with Soil Plant Analysis Development (SPAD) indicated the suitability of the color models RGB, rgb, and DGCI-rgb. Among the linear regression models, the models based on mean brightness ratio (rgb) alone or in combination with a dark green color index (DGCI-rgb) show a good correlation between the predicted Chl content and relative Chl content. A feed-forward backpropagation-type network was also developed following the optimization of hidden neurons, training, and transfer functions. The predicted Chl contents showed a good correlation with SPAD values. Compared to the linear regression model, the developed artificial neural network model was found to be more efficient in predicting the Chl content, particularly with RGB index.
\end{abstract}

Additional key words: color index; Oryza sativa; RGB color space.

\section{Introduction}

Chlorophyll (Chl) is the most important pigment molecule that absorbs light and subsequently transfers its energy to drive the photochemical reactions of photosynthesis. The contents of Chl $a$ and $b$ are directly related to the photosynthetic ability and primary productivity of plants. It can also reflect the physiological status and is considered to be an important indicator for plant health status (Curran et al. 1990, Pagola et al. 2009, Yadav et al. 2010, MunozHuerta et al. 2013). Chl absorbs both red (625-675 nm) and blue (425-475 $\mathrm{nm})$ light, whereas the wavelength corresponding to green color $(520-560 \mathrm{~nm})$ is poorly absorbed and leaves are green as they reflect more this color (Goetz et al. 1983, Rigon et al. 2012). The Chl content of leaves further serves as an indicator of nitrogen status (Yuan et al. 2016, Ravier et al. 2017) and additional information on plant nutrient status (Steele et al. 2008, Sim et al. 2015, Rigon et al. 2016). A good correlation between $\mathrm{Chl}$ and nitrogen content has been observed in leaf tissue from a variety of plant species, such as rice (Lin et al. 2010, Wang et al. 2014, Yuan et al. 2016), tall fescue (Errecart et al. 2012), and wheat (Ravier et al. 2017).

Compared to conventional destructive spectroscopic methods of $\mathrm{Chl}$ estimation (Lichtenthaler and Wellburn
1983), sensor-based, modern, noninvasive approaches have received considerable attention due to their highthroughput and real-time measurement abilities (Cassol et al. 2008, Yadav et al. 2010, Rigon et al. 2012, Stefan et al. 2013, Hu et al. 2014, Jinwen 2014, Novichonok et al. 2016). Different types of Chl meters (Pagola et al. 2009, Novichonok et al. 2016), multi-spectral (Reyniers et al. 2006) and hyper-spectral sensors (Chen et al. 2010, Li et al. 2014) have been developed to measure absorbance and spectral reflectance of leaves. Among Chl meters, the SPAD meter (SPAD-502, Minolta, Japan) has been widely used to measure the $\mathrm{Chl}$ content as well as to assess crop nitrogen status nondestructively (Uddling et al. 2007, Cabangon et al. 2011, Ling et al. 2011, Liu et al. 2012, Wang et al. 2013, 2014; Yuan et al. 2016, Vesali et al. 2017, Agarwal and Dutta Gupta 2018). The instrument measures transmission of light absorbed by $\mathrm{Chl}$ in the red band $(650 \mathrm{~nm})$, as well as transmission of infrared light $(950 \mathrm{~nm})$, where no absorption of Chl occurs. The calculation of a SPAD value by the instrument is based on the ratio of transmitted red light and infrared light, and the SPAD value was found to be well correlated with the Chl content (Markwell et al. 1995). Other nondestructive techniques, based on satellite or airborne spectral and hyperspectral reflectance imaging, have been developed

$\overline{\text { Received }} 10$ May 2018, accepted 22 October 2018.

${ }^{+}$Corresponding author; e-mail: $\underline{s d g @ a g f e . i i t k g p . a c . i n ~}$

Abbreviations: ANN - artificial neural network; Chl - chlorophyll; DGCI - dark green color index; HSV - hue, saturation, and value color space; MAE - mean absolute error; RGB - red, green, and blue color space; RMSE - root mean square error; SPAD - soil plant analysis development; Train LM - Levenberg-Marquardt backpropagation; VI - vegetation index.

Acknowledgements: The authors wish to thank Dr. N.S. Raghuwanshi for allowing us to capture the leaf images from their experimental rice field. We wish to thank Mr. A. Agarwal for his help in modifying the figures and two anonymous reviewers for their very helpful comments on previous draft. 
in the recent past to measure the nitrogen status of plants. These techniques are costly and usually implemented by satellite-mounted sensors and their applications have been restricted due to infrequent satellite overpasses, clouds, and background soil interferences at farmer level. Among the nondestructive methods, the SPAD and related Chl meter is cheap, user friendly, and does not suffer from any background interferences. However, a large number of repetitions of SPAD measurement is needed due to the small sampling area $\left(6 \mathrm{~mm}^{2}\right)$ of the instrument. In recent years, digital cameras with embedded sensors have been utilized as a low cost instrument for estimation of the Chl content. Digital imaging together with image segmentation and RGB (red, green, blue) color space model is now becoming a potential approach for smart agriculture; capable of estimating $\mathrm{Chl}$ contents, biomass, nitrogen content, and leaf area index (Dutta Gupta et al. 2013, Riccardi et al. 2014, Wang et al. 2014, Rigon et al. 2016). Relationships between indices of spectral reflectance and Chl absorbance with RGB image components have indicated the potential of digital image segmentation in RGB components to estimate the Chl content (Santos do Amaral et al. 2018). Variations in light conditions can affect imaging constraints due to different ambient light conditions. Shadows on leaves have been addressed by several workers utilizing various types of color indices or chromatic transformations. Several studies reported the capability of the dark green color index (DGCI) to determine the nitrogen status of plants. This index was introduced by Karchar and Richardson (2003) utilizing HSB (hue, saturation, and brightness) color space. The DGCI showed a good correlation with the nitrogen content in corn (Rorie et al. 2011a,b) Apart from this, other indices derived from images have been also found useful in reducing the effect of lighting variation. Wang et al. (2013) used $\mathrm{G}-\mathrm{R}$ (difference between green component and red component), G/R (green divided by red), $\mathrm{R} /(\mathrm{R}+\mathrm{G}+\mathrm{B}$ ) (normalized red index, NRI), G/(R+G + B) (normalized green index, NGI) and hue to estimate biomass and leaf area index along with nitrogen content.

The recent advancements in smartphones with highspeed processors and camera sensors offer an opportunity to utilize their image acquisition as well as processing abilities for applications in many areas including agriculture (Gomez-Robledo et al. 2013, Pongnumkul et al. 2015, Friedrichs et al. 2017). Smartphone images have been utilized to measure a citrus yield (Gong et al. 2013), crop water requirements (Confalonieri et al. 2013), phosphorus content in soil (Moonrungsee et al. 2015), and to estimate Chl contents in corn (Vesali et al. 2015, 2017) and soybean plants (Rigon et al. 2016). A recent study demonstrated its capability in measuring Chl fluorescence (Friedrichs et al. 2017). However, variations exist between the color models and the proposed regressions that fit well with SPAD values among the findings. Moreover, in most of studies, analysis was based on linear relationships between the color indices and SPAD-derived Chl contents. The nonlinear and nondeterministic nature of a biological system makes such a relationship incomprehensible. Artificial neural network (ANN) modeling has an inherent ability in determining the complex nonlinear relationships between the input and output of a biological system based on the strengths of their interconnected neurons (Krogh 2008, Prasad and Dutta Gupta 2008, Osama et al. 2015). Feed Forward Neural Network (FFNN) with a backpropagation (BP) algorithm was developed to estimate Chl contents from hyperspectral data (Liu et al. 2010) and in one instance from smartphone contact image (Vesali et al. 2015).

The present work describes the imaging of rice leaves using a smartphone under natural light and subsequent modeling approach to retrieve leaf $\mathrm{Chl}$ contents of rice using various RGB color indices.

\section{Materials and methods}

Plant material and experimental site: The data were collected from rice (Oryza sativa L. cv. IR36) plantations established at the Agricultural and Food Engineering Farm, Indian Institute of Technology Kharagpur, India, with three different fertilizer treatments designated as $U$ 60, $\mathrm{U} 90$, and $\mathrm{U} 120$ representing 27.6, 41.4, and $55.2 \mathrm{~kg}(\mathrm{~N})$ $\mathrm{ha}^{-1}$, respectively. The site plot is in agro-climatic zone WB-5 (undulating red and laterite zone of West Bengal) of India with an average rainfall of $8.5 \mathrm{~mm}$ at the time of imaging. The type of soil present in the experimental plots is sandy clay loam in nature. The traditional practices were followed for the crop management. A representative experimental plot is shown in Fig. 1S (supplement).

Imaging of rice leaves using a smartphone: The images of rice leaves were acquired from the experimental plot at the end of the tillering stage (around $30 \mathrm{~d}$ after transplanting). Fifty rice plants were randomly selected from each experimental site and the fourth leaf on the main stem of each plant was used for image acquisition. The leaf mid portion was considered as the target during image acquisition. The images of plant leaves were captured by a YU Yureka ao5510 (Micromax, India), 13 mega pixel smartphone camera consisting of Sony IMX135 CMOS image sensor with auto-focus feature. A magenta sheet was used behind the leaf to eliminate the field background in the leaf image according to Vollmann et al. (2011). Leaf images were captured by holding the smartphone parallel to the leaf surface, with the aperture at a fixed distance of $12 \mathrm{~cm}$ from the leaf. The camera was set at aperture of $\mathrm{f} / 2.2$ with auto-mode for ISO and exposure time with the flash turned off. The images were captured between 9:00 and 12:30 h IST on sunny days at a mean temperature of $32-34^{\circ} \mathrm{C}$ and precipitation of $0-1 \mathrm{~mm}$. They were saved in JPEG format (joint photographic experts group). Altogether, there were 50 digital images for each nitrogen treatment.

SPAD measurement for estimation of relative Chl content: SPAD values were simultaneously measured from the respective leaves by using a SPAD-502 chlorophyll meter (Minolta, Japan). Five SPAD values were obtained from the same area of rice leaf which was selected for image acquisition and the mean SPAD value (relative Chl 
content) was calculated for each leaf. The SPAD value has an exponential relation with $\mathrm{Chl}$ content of plants measured spectrophotometrically:

$[\mathrm{Chl}]=10^{\mathrm{M}^{\wedge} 0.265}$

where [Chl] is the Chl content in unit of $\mu \mathrm{mol} \mathrm{m}{ }^{-2}$ and $\mathrm{M}$ is the SPAD value (Markwell et al. 1995).

Image preprocessing, segmentation and feature extraction: Image preprocessing was done using Adobe Photoshop 7.0 (Adobe Systems Inc., USA) to compensate the variations in field light conditions during the course of image acquisition. The images were subjected to white-balancing using a white to black gradient strip as a reference (Photo Blog Stop, accessed on 26 August 2016, http://photoblogstop.com/photoshop/accurate-whitebalance-adjustments-in-photoshop). A $50 \%$ gray point was selected from the white to black gradient strip placed right side to the leaf during image acquisition. With the help of the selected $50 \%$ gray point the image was white-balanced to ensure image capture independent of light variation. A Matlab ${ }^{\circledR} 8.1$ (MathWorks Inc., Natick, USA) based program was developed for image segmentation, RGB and HSV features extraction using a binary threshold concept in which the threshold values were chosen from minimum and maximum RGB values possible for imparting various shades of green color in the leaves (Pound and French 2014). The threshold values for $R, G$, and $B$ were $0-130$, 51-255, and 0-130, respectively (RapidTables, http:// www.rapidtables.com/web/color/RGB Color.htm). The program selected pixels from the leaf images having their RGB values within the stipulated threshold (Fig. 2S, supplement). Since the RGB values of the magenta background are lying outside the specified thresholds, the background was eliminated during image segmentation. Finally, the developed Matlab ${ }^{\circledR}$ code extracted the mean RGB and HSV features of the leaf from the respective digital image.

Derivation of color indices: The color images captured by a smartphone are composed of pixels consisting of RGB values ranging between 0 and 255 for each band $R, G$, and B. R, G, and B are the mean values of red, green, and blue components of an image and each RGB color pixel represents a depth of 24 bit. RGB values were normalized to obtain the mean brightness ratio (rgb) of each color. From HSV color space, the hue $(\mathrm{H})$, saturation $(\mathrm{S})$, and value (V) components of each image and $\mathrm{Y}, \mathrm{Cb}$, and $\mathrm{Cr}$ attributes of $\mathrm{YCbCr}$ color space were extracted (Table 1S, supplement). Apart from these main channel color spaces, other combinations of color indices, i.e., $\mathrm{G}-\mathrm{B}, \mathrm{G}-\mathrm{R}$, and $\mathrm{G} / \mathrm{R}, \mathrm{VI}_{\text {green }}[(\mathrm{G}-\mathrm{R}) /(\mathrm{G}+\mathrm{R})]$, and DGCI $([($ Hue $/ 60-1)+$ $(1-\mathrm{S})+(1-\mathrm{B})] / 3)$ were also extracted from RGB values. The equations used to derive the various color indices are presented in Table $1 \mathrm{~S}$.

Correlation of color indices with relative $\mathrm{Chl}$ content (SPAD) values: The mean value of $R, G, B$ and different color indices (as in detail in the Table S1, supplement) were correlated with SPAD values following the Pearson's correlation analysis using Microsoft Office Excel 12.0 Data Analysis Tool Pack (Microsoft, Washington, USA).

Multiple linear-regression modeling to estimate $\mathrm{Chl}$ content from leaf image features: A linear-regression analysis was performed using dataset comprised of 50 leaves with a U 60 treatment using Matlab ${ }^{\circledR} 8.1$ (MathWorks Inc., Natick, USA) for the prediction of the Chl content from RGB color space model as described in Yadav et al. (2010). A similar approach was also adopted to predict the Chl content using the values obtained from the expression $([(\mathrm{Hue} / 60-1)+(1-\mathrm{S})+(1-\mathrm{B})] / 3)$ and $\mathrm{rgb}$, hereafter referred to as DGCI-rgb linear regression model. The selection of type of models was based on the analysis of correlation between the color indices and SPAD values. The model equations used are:

$$
\begin{aligned}
& {[\mathrm{Chl}]_{\mathrm{P} 1}=\mathrm{a}_{1} * \mathrm{R}+\mathrm{b}_{1} * \mathrm{G}+\mathrm{c}_{1} * \mathrm{~B}} \\
& {[\mathrm{Chl}]_{\mathrm{P} 2}=\mathrm{a}_{2} * \mathrm{r}+\mathrm{b}_{2} * \mathrm{~g}+\mathrm{c}_{2} * \mathrm{~b}} \\
& {[\mathrm{Chl}]_{\mathrm{P} 3}=\mathrm{a}_{3} * \mathrm{DGCI}+\mathrm{b}_{3} * \mathrm{r}+\mathrm{c}_{3} * \mathrm{~g}+\mathrm{d}_{3} * \mathrm{~b}}
\end{aligned}
$$

for RGB, rgb, and DGCI-rgb models, respectively. Chl 1 , $\mathrm{Chl}_{\mathrm{P} 2}$, and $\mathrm{Chl}_{\mathrm{P} 3}$ are the predicted $\mathrm{Chl}$ contents. R, G, and $B$ represent mean reflectance value, whereas $r, g$, and $b$ are the mean reflectance ratio of each primary color. The coefficients preceding the color index variables are the model parameters which were determined using the matrices:

$$
\begin{aligned}
& {\left[\mathrm{a}_{1} \mathrm{~b}_{1} \mathrm{c}_{1}\right]^{\mathrm{T}}=\left[\mathrm{A}^{\mathrm{T}}{ }_{\mathrm{RGB}} * \mathrm{~A}_{\mathrm{RGB}}\right]^{-1} * \mathrm{~A}^{\mathrm{T}}{ }_{\text {RGB }} * \mathrm{Y}} \\
& {\left[\mathrm{a}_{2} \mathrm{~b}_{2} \mathrm{c}_{2}\right]^{\mathrm{T}}=\left[\mathrm{A}^{\mathrm{T}}{ }_{\mathrm{rb}} * \mathrm{~A}_{\mathrm{rgb}}\right]^{-1} * \mathrm{~A}_{\text {rgb }}^{\mathrm{T}} * \mathrm{Y}} \\
& {\left[\mathrm{a}_{3} \mathrm{~b}_{3} \mathrm{c}_{3} \mathrm{~d}_{3}\right]^{\mathrm{T}}=\left[\mathrm{A}^{\mathrm{T}}{ }_{\text {DGCI-rgb }} * \mathrm{~A}_{\text {DGCI-rgb }}\right]^{-1} * \mathrm{~A}_{\text {DGCI-rgb }}^{\mathrm{T}} * \mathrm{Y}}
\end{aligned}
$$

where $A_{R G B}$ is the mean brightness value of primary colors and $\mathrm{A}_{\mathrm{rgb}}$ and $\mathrm{A}_{\mathrm{DGCl}-\mathrm{rgb}}$ represent the mean brightness ratio of primary colors and image feature DGCI with mean brightness ratio. $\mathrm{T}$ denotes the transpose. Vector $\mathrm{Y}$ represents the relative $\mathrm{Chl}$ content of the leaves determined by SPAD meter. The relation between the model predicted and SPAD measured Chl content was assessed by coefficient of determination $\left(R^{2}\right)$ and root mean square error (RMSE). The RMSE is expressed as:

RMSE $=\sqrt{\frac{\sum_{i=1}^{n}\left(x_{i}-x_{i}\right)^{2}}{n}}$

where $\mathrm{X}_{\mathrm{i}}$ is the relative Chl content, $\mathrm{x}_{\mathrm{i}}$ is the predicted $\mathrm{Chl}$ content and $\mathrm{n}$ is the number of measurements.

Validation of linear-regression model was done by using the datasets obtained from 50 leaves of both U 90 and $U$ 120 treated rice plants. The model equation and the model parameters as derived from U 60 dataset were considered. The RGB, rgb, and DGCI-rgb values of the U 90 and U 120 datasets are substituted in the respective model equations and the predicted $\mathrm{Chl}$ content values thus generated were correlated with the SPAD measured relative $\mathrm{Chl}$ content values of the corresponding leaves of U 90 and U 120 treatments.

ANN modeling for prediction of Chl content from leaf images: For noninvasive prediction of $\mathrm{Chl}$ content, 
a Feed Forward Neural Network with a backpropagation (BP) algorithm (FFNN-BP) ANN model was developed using the Matlab ${ }^{\circledR} 8.1$ platform. FFNN-BP is a multilayer perceptron training algorithm in which the input values are fed forward to obtain a nonlinear relationship between the input (RGB, rgb, DGCI-rgb) and output (Chl content) variables, further error is calculated and back propagated through the network during training for updating weights in the nonlinear relationship generated by the network (Krogh 2008, Živković et al. 2009). The performance function (mean squared error) was derived following the comparison of the predicted properties with the observed properties of the process. The RGB model network topology is depicted in Fig. 1. However, there were four input nodes for DGCI-rgb model. The dataset of U 60 treatment was divided into two groups. The training set consisted of data from 35 leaves, whereas the data obtained from 15 leaves were used for testing the ANN model. A code was created in Matlab for the RGB, rgb, and DGCI$\mathrm{rgb}$ models and for training the ANN model as well as predicting the $\mathrm{Chl}$ content from the test dataset. The model uses the logarithmic sigmoid transfer function for both the hidden and output layer neurons which scales the input data into an open interval of $0-1$. The input variables were normalized by using the expression:

$\mathrm{x}_{\mathrm{N}}=\frac{\mathrm{x}-\mathrm{x}_{\min }}{\mathrm{x}_{\max }-\mathrm{x}_{\min }}$

where $X_{N}, X, X_{\max }$, and $X_{\min }$ are the normalized, real, maximum, and minimum values of the input variables, respectively.

Initially, 11 networks were tested with 11 training functions, i.e., Train GDA, Train GDX (gradient descent

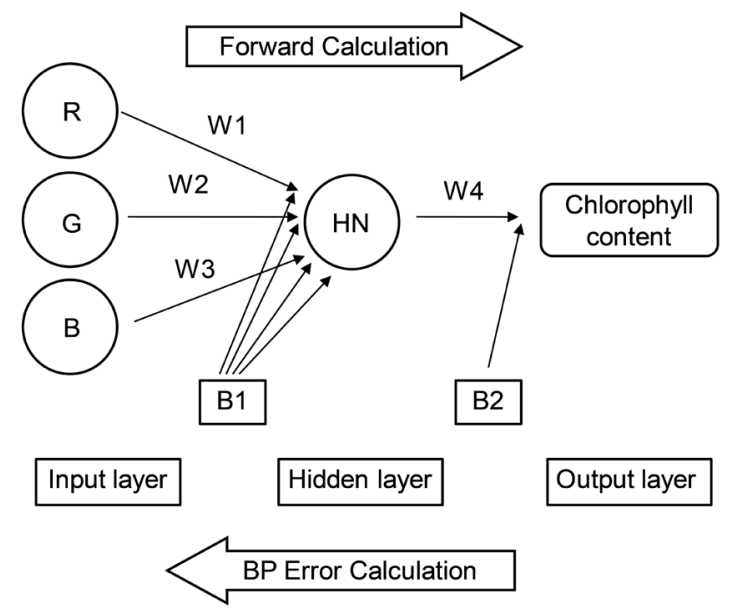

Fig. 1. Artificial neural network (ANN) architecture for RGB model with 3-1-1 structure.

with momentum and adaptive learning rate), Train GDM (gradient descent with momentum backpropagation), Train RP (resilient backpropagation), Train BR (Bayesian regularization backpropagation), Train CGB (conjugate gradient backpropagation), Train CGF (conjugate gradient backpropagation with Fletcher-Reeves updates), Train OSS (one-step secant backpropagation), Train
SCG (scaled conjugate backpropagation), Train BFG (BFGS-quasi Newton backpropagation), and Train LM (Levenberg-Marquardt backpropagation) following the inbuilt command of Matlab. The training function which performed best was selected on the basis of a maximum $R^{2}$, least RMSE, and least average percentage difference (APD) during testing.

The average percentage difference (APD) was calculated as follows:

$\mathrm{APD}=\frac{\sum_{\mathrm{i}=1}^{\mathrm{N}}\left(\mid \frac{\mathbf{E} 1_{\text {predicted }}-\mathbf{K} \mathbf{1}_{\text {relative }}}{\left.\mathrm{Chl_{ \text {relative } }} \mid\right) \cdot 100}\right.}{\mathrm{N}}$

where $\mathrm{Chl}_{\text {predicted }}=$ predicted $\mathrm{Chl}$ content, $\mathrm{Chl}_{\text {relative }}=$ relative Chl content, $\mathrm{N}=$ number of measurements.

The parameters optimized for the ANN models are mentioned below:

$\begin{array}{ll}\text { Training function } & \text { Train LM (Levenberg-Marquardt) } \\ \text { Transfer function } & \text { LOGSIG } \\ \text { Performance function } & \text { MSE } \\ \text { Hidden nodes } & 1 \\ \text { Hidden layers } & 1 \\ \text { Target epochs } & 500 \\ \text { Target error } & 0.0001 \\ \text { Learning rate } & 0.01\end{array}$

For the validation of the ANN model the datasets of $\mathrm{U} 90$ and $\mathrm{U} 120$ were fed into the trained network as obtained from U 60 treatment. Thus, model validation was performed with a test set of 50 leaves each from U 90 and U 120 treatment instead of test set of 15 leaves from U 60 treatment during model development.

Performance evaluation of the models: The performance of the linear and ANN models in predicting the Chl content was assessed by comparing the RMSE and mean absolute error (MAE). The MAE is expressed as

MAE $=\frac{1}{n} \sum_{i=1}^{n}\left|S_{i}-P_{i}\right|$

where $S_{i}$ and $P_{i}$ are the SPAD and predicted Chl values, $n$ is the number of observations. The accuracy of the models with respect to the benchmark of the ability of prediction was also evaluated by percent bias (PBias) and is expressed as:

$$
\text { PBias }=\frac{\sum_{\mathrm{i}=1}^{\mathrm{n}}\left(\mathrm{P}_{\mathrm{i}}-\mathrm{S}_{\mathrm{i}}\right)}{\sum_{\mathrm{i}=1}^{\mathrm{n}}\left(\mathrm{S}_{\mathrm{i}}\right)} \times 100
$$

where $S_{i}$ and $P_{i}$ represent SPAD and predicted Chl values and $\mathrm{n}$ is the number of observations.

The overall steps of image analysis system including the modeling approach for noninvasive estimation of leaf $\mathrm{Chl}$ index of rice under field conditions using a smartphone are shown in Fig. 2. 


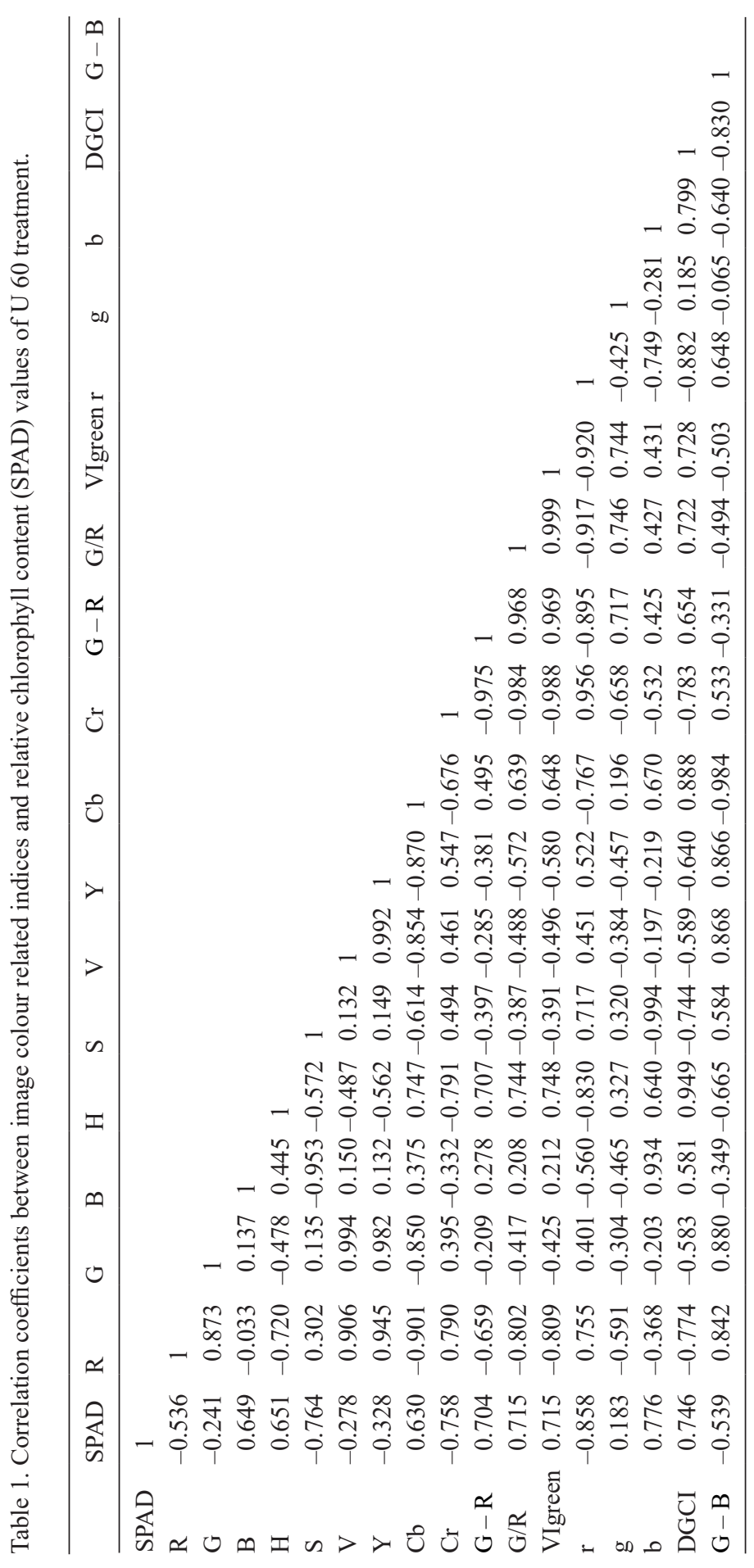

\section{Results}

Correlation of image-based color indices with SPAD measured relative Chl content: Pearson's correlation analysis was performed to find correlation between the color index and the relative $\mathrm{Chl}$ content with the datasets of $\mathrm{U} 60, \mathrm{U} 90$, and U 120 treatments (Tables 1, 2). A negative correlation with the relative $\mathrm{Chl}$ content was found with color indices R, G, G - B, r, S, V, Y, and Cr, whereas

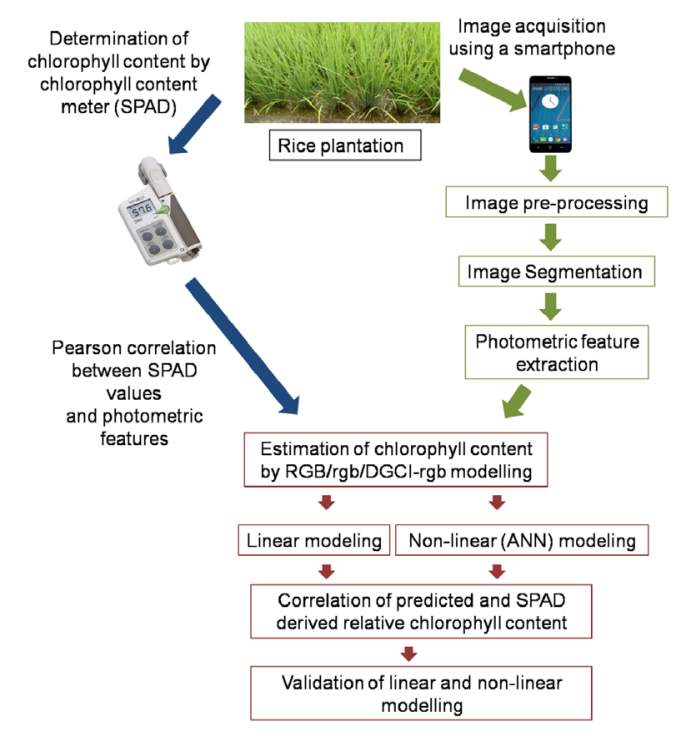

Fig. 2. A digital imaging system for predicting the leaf chlorophyll content of rice in a noninvasive manner under field condition using a smartphone. indices $\mathrm{B}, \mathrm{b}, \mathrm{G}-\mathrm{R}, \mathrm{G} / \mathrm{R}, \mathrm{Cb}, \mathrm{H}$ were positively correlated. The strongest linear relationship $\left(\mathrm{R}^{2}=-0.858,-0.748\right.$, and -0.728 , respectively, for $\mathrm{U} 60, \mathrm{U} 90$, and $\mathrm{U} 120$ ) with SPAD value was obtained with $r$ in the RGB color space model. Color index $\mathrm{G}$ was poorly correlated with the Chl content. Considering the relationships prevalent in this study, modeling was performed with the color models RGB, rgb, and DGCI-rgb. 


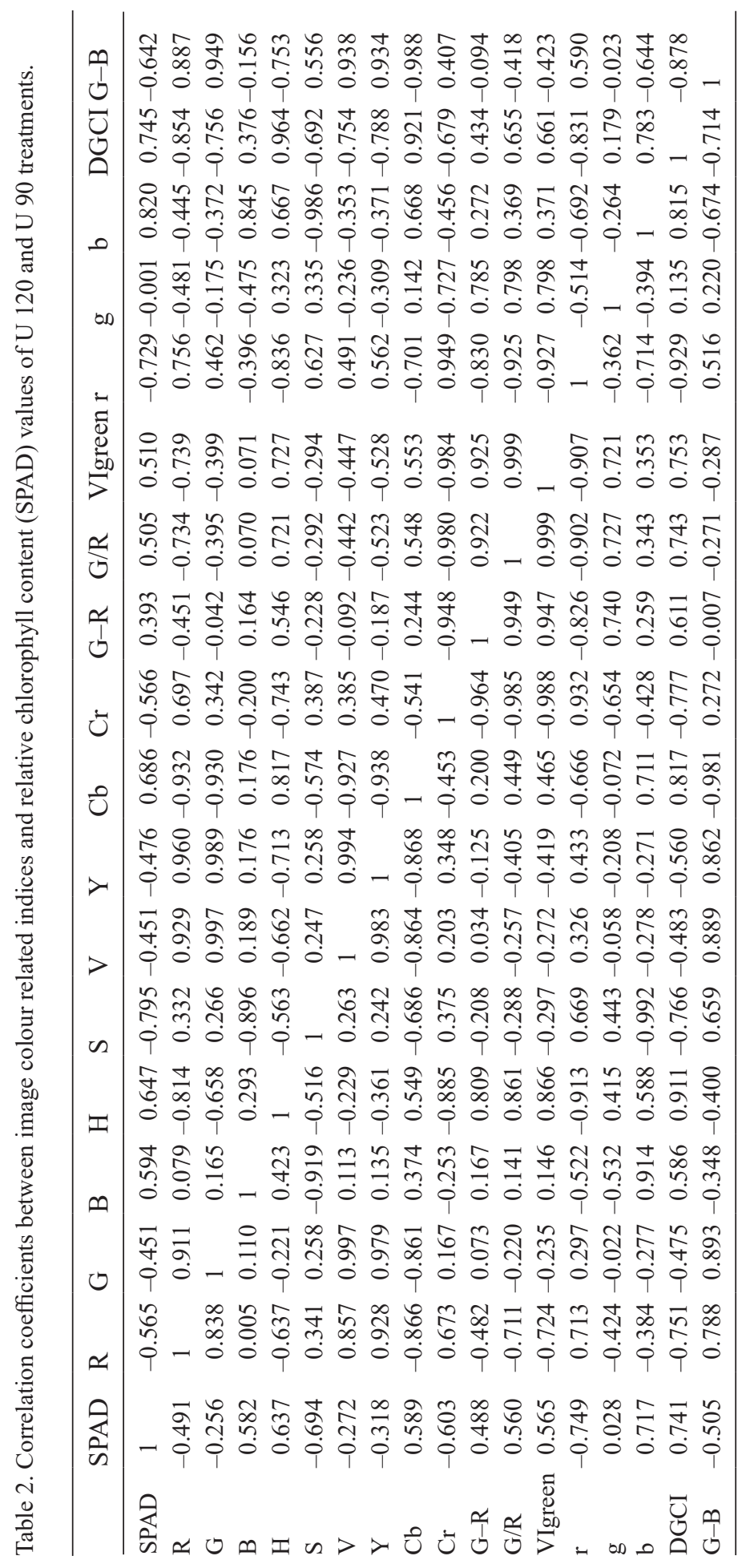

Linear modeling for prediction of Chl content and model validation: Linear regression models were developed for $\mathrm{RGB}, \mathrm{rgb}$, and DGCI-rgb datasets corresponding to U 60 treatment using Matlab ${ }^{\circledR}$ 8.1. The model parameters calculated were $\mathrm{a}_{1}=-0.4998, \mathrm{~b}_{1}=0.7917, \mathrm{c}_{1}=0.3794 ; \mathrm{a}_{2}=$ $-6.8731, \mathrm{~b}_{2}=58.2756, \mathrm{c}_{3}=93.8636$; and $\mathrm{a}_{3}=-17.8162$, $\mathrm{b}_{3}=-12.9857, \mathrm{c}_{3}=72.3768, \mathrm{~d}_{3}=118.4315$, respectively, for RGB, rgb, and DGCI-rgb models. The performance of the models in terms of $R^{2}$ and RMSE is shown in Fig. 3. A poor correlation $\left(R^{2}=0.335\right)$ between model predicted and SPAD-derived Chl content was observed with RGB model, whereas rgb and DGCI-rgb models predicted Chl content with significant correlations of $R^{2}=0.776$ and $R^{2}=0.792$, respectively.

The linear regression model developed was validated using the experimental dataset of U 120 and U 90 treat- 


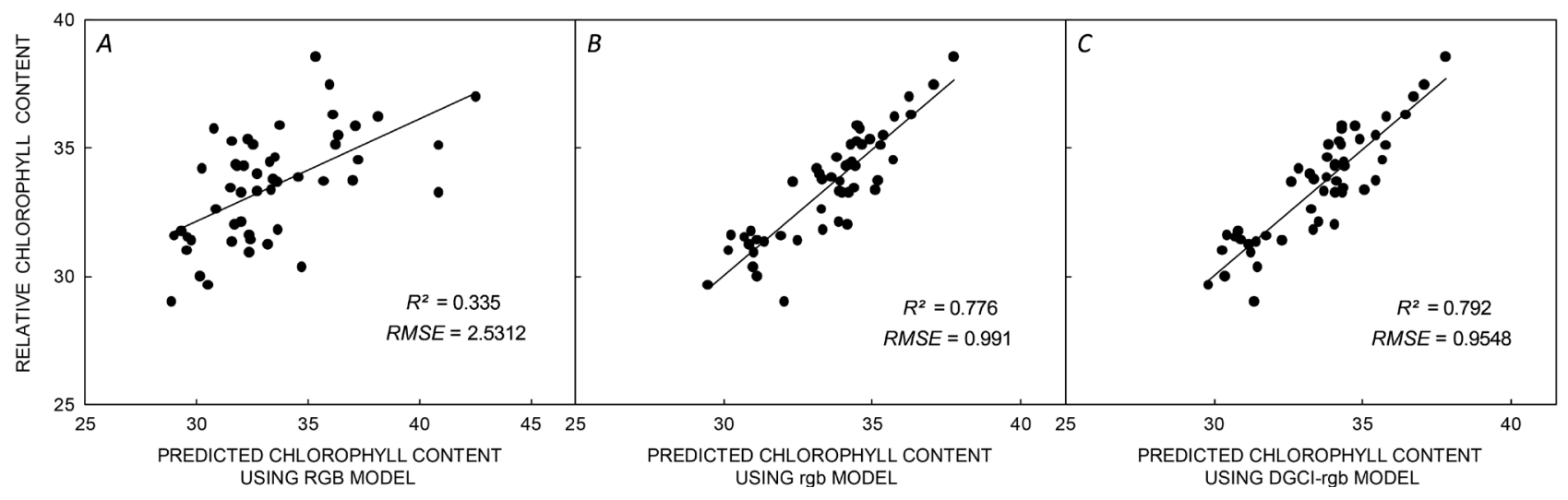

Fig. 3. The correlation of chlorophyll (Chl) content predicted by linear RGB $(A), \operatorname{rgb}(B)$, and DGCI-rgb $(C)$ models to that of relative Chl content from U 60 dataset.

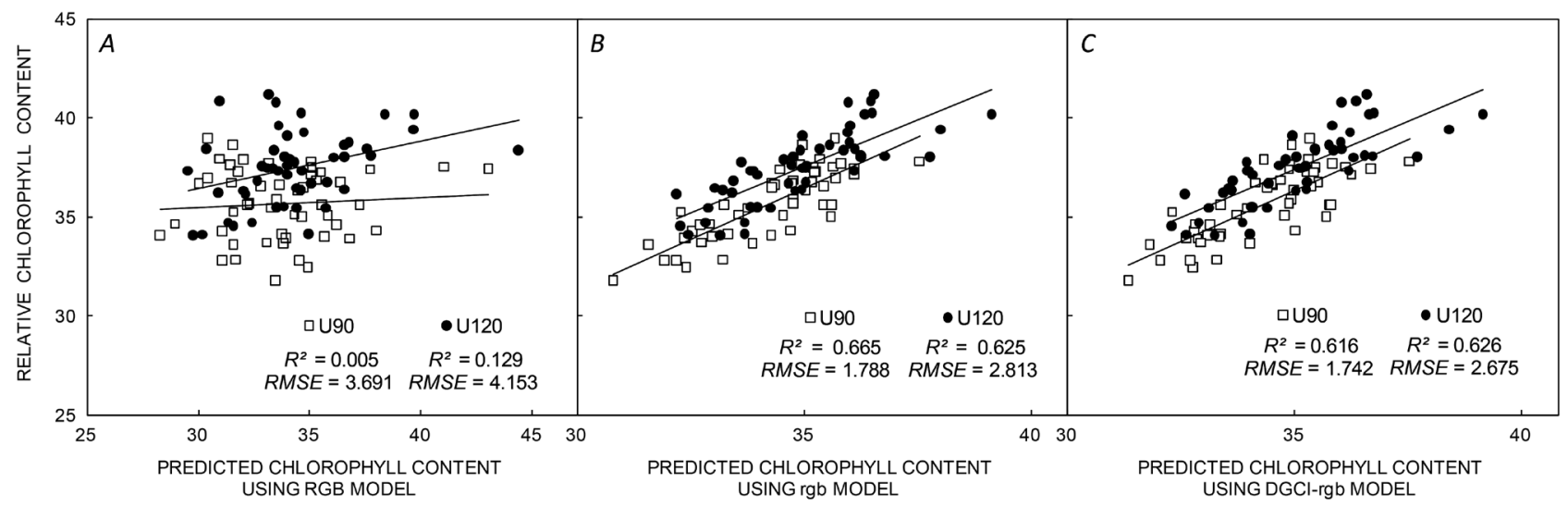

Fig. 4. Validation of the linear RGB $(A), \operatorname{rgb}(B)$, and DGCI-rgb $(C)$ models using datasets of $\mathrm{U} 90$ and $\mathrm{U} 120$ and their ability to predict chlorophyll content.

ments. The predicted Chl content generated from both the datasets using RGB linear model showed poor correlation with SPAD measured relative Chl content. However, rgb and DGCI-rgb models were capable of predicting the Chl content efficiently having high correlation with SPAD value (Fig. 4).

ANN modeling for estimation of relative Chl content: For the FFNN-BP model, the input variables RGB, rgb, DGCI-rgb having one hidden layer with one node were trained with datasets comprised of 35 leaves and were tested with a dataset of 15 leaves with a $U 60$ treatment. Initially, the model development was tested with different network architectures, network internal parameters, and various training functions. The optimum number of hidden neurons was selected on the basis of high $R^{2}$ and low RMSE values. The effective network topology for RGB model was observed to be $3-1-1$, where 3 represents the input variables, 1 denotes the hidden neuron layer, and 1 represents the output as shown in Fig. 1. During selection of the optimum number of hidden neurons, a peaking effect was observed as the error increased with an increasing number of neurons beyond one. A significant influence of the training function in optimization of the ANN modeling was observed. The optimal efficiency of the ANN networks was obtained by comparing the model predicted values with the experimental data obtained from the SPAD meter. A comparative assessment between the measured $\mathrm{Chl}$ content and all trained network output data for the least deviation from the target range revealed that the output range of network 'Trainlm' was closest to the actual data. Among the 11 training functions, 'Train LM' resulted in the lowest RMSE of $2.91, R^{2}$ value of 0.797 , and APD of 2.696 (Fig. 3S, supplement), and hence, was selected for use in the modeling approach to predict the Chl content. Fig. 5 shows the correlation between the predicted and SPAD-measured Chl contents for RGB, rgb, and DGCI-rgb models. All the models exhibited a significant correlation between the predicted and experimental $\mathrm{Chl}$ content with $R^{2}$ values of $0.797,0.806$, and 0.807 , respectively, for RGB, $\mathrm{rgb}$, and DGCI-rgb parameters. Further, the efficiency of the developed models was validated with the selected image features of test datasets obtained from U 90 and $\mathrm{U} 120$ treatments. The predicted Chl contents thus gene- 


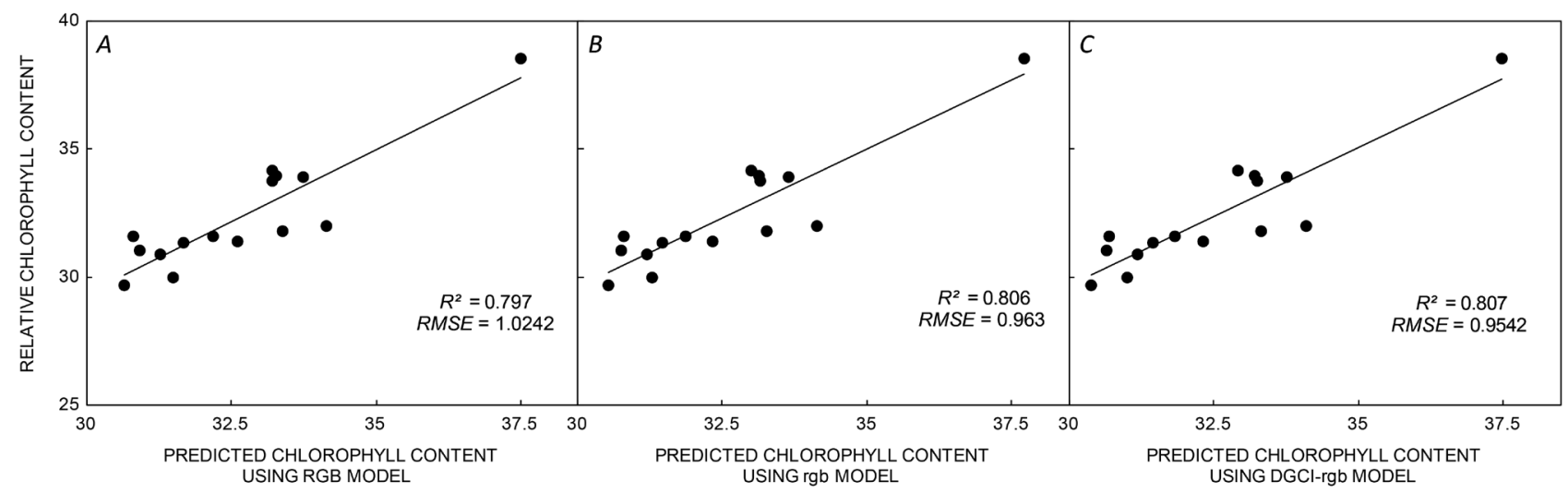

Fig. 5. Relationship between chlorophyll (Chl) content predicted by RGB (A), rgb (B), and DGCI-rgb $(C)$ artificial neural network models to that of relative $\mathrm{Chl}$ content (SPAD values) using the dataset of $\mathrm{U} 60$.

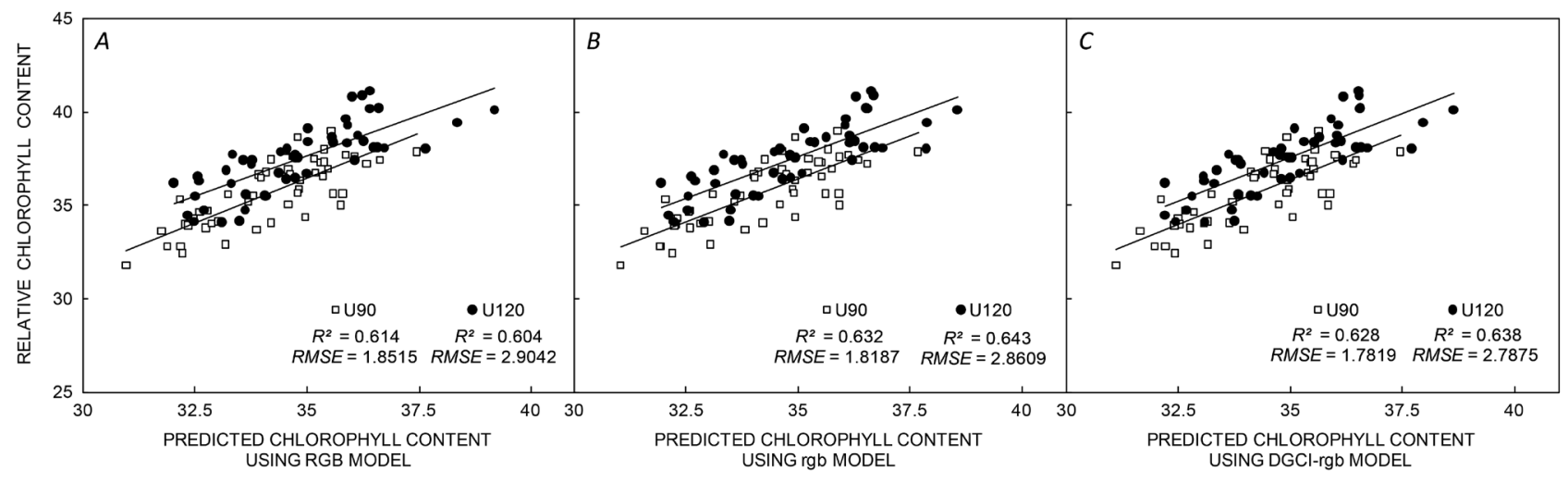

Fig. 6. Validation of the RGB $(A), \operatorname{rgb}(B)$, and DGCI-rgb $(C)$ artificial neural network models using the test datasets of $\mathrm{U} 90$ and $\mathrm{U} 120$ and their ability to predict chlorophyll content.

rated showed a significant correlation with SPAD values for all the three models (Fig. 6).

Performance of the developed models to predict relative Chl content: The quantitative relationships between the model-predicted and SPAD-derived Chl content in rice during performance evaluation of the developed RGB, rgb, and DGCI-rgb models from the test datasets of $\mathrm{U} 90$ and $\mathrm{U} 120$ are summarized in Tables $2 \mathrm{~S}$ and $3 \mathrm{~S}$ (supplements). The ANN model performed better than the linear model particularly with the RGB color space. With the rest of the color indices, both the linear and nonlinear model performed equally well. The predicting ability of the developed models was also tested with percent bias. The PBias values indicate that the predicted values from both the models are within the acceptable limit of $< \pm 10 \%$.

\section{Discussion}

Correlation of color indices with relative $\mathrm{Chl}$ content: In recent years, the successful application of digital image processing with color-related indices has opened up a new vista to estimate variables of agronomic importance. The present study demonstrated the ability of index ' $b$ ' in the RGB color space to predict $\mathrm{Chl}$ content with its best fit to SPAD value, whereas the green parameter $(\mathrm{G})$ was found to be ineffective. Similar relationship of color indices with SPAD measurement was also observed in corn following image acquisition using spectral absorption photometry (SAP) and light aided spectral absorption photometry (LASAP, Vesali et al. 2017). In contrast, a good relationship of $\mathrm{Chl}$ content with the single color component index ' $G$ ' was observed in soybean (Rigon et al. 2016). It has been pointed out that the RGB color space does not always represent properly the green value of vegetation. A conversion of RGB values has been suggested to more intuitive HSV color model to represent the correlation of color index with SPAD value (Karcher and Richardson 2003). In the present study, the index 'S' of the HSV color model had strong linear relation with the Chl content. Among the indices of HSV color spectrum, DGCI showed a significant correlation with SPAD value in all the treatments. This has been the most widely used color model among the various image-related indices that 
fitted well to Chl contents (Rorie et al. 2011a, Lee and Lee 2013). However, in their work with corn, Vesali et al. $(2015,2017)$ reported poor correlation of DGCI with the value of SPAD. Differences in the species studied, image acquisition methods with type of sensors utilized, irradiation conditions, and operating system of the camera may account for such variations in the relationship (Pagola et al. 2009, Wang et al. 2013, Rigon et al. 2016). The intrinsic characteristics of plant leaves, such as nature of cuticle, presence or absence of trichomes, and leaf anatomical features, may regulate the reflectance pattern and consequently change the relationship. Considering the relationships prevalent in this study, modeling was performed with the color models RGB, rgb, and DGCI-rgb. The developed image segmentation algorithm is capable of extracting the RGB features under field conditions without any additional device fitted to the smartphone. It makes the image acquisition user friendly along with bypassing the constraints of lighting variations in real conditions compared to the contact imaging and LASAP methods adopted by Vesali et al. (2017).

Linear modeling to predict $\mathrm{Chl}$ content and model validation: In most of studies, the relationship between color indices and SPAD values has been evaluated according to the simple correlation and regression analysis (Wang et al. 2014, Rigon et al. 2016, Vesali et al. 2017). The regression model developed in our study was able to establish a best fit to the SPAD-measured data and was also capable to predict $\mathrm{Chl}$ contents efficiently. The relationship obtained is in agreement with the findings of Yadav et al. (2010), Dutta Gupta et al. (2014), and Dutta Gupta and Pattanayak (2017), where linear regression models were used and a rgb model was found suitable for the prediction of $\mathrm{Chl}$ content in micropropagated potato. The similarity in the predicting ability between the model calibration and validation suggests the robustness of the imaging method and the developed model. Moreover, similar trends in the values of $\mathrm{R}^{2}$ and RMSE for different nitrogen treatments indicated the consistency of model performance at different nitrogen concentrations.

\section{ANN modeling for estimation of relative Chl content:} Despite the successful application of linear regression model, such modeling approach suffers from poor predicting ability in a nonlinear biological system. The intrinsic nondeterministic behavior of the biological samples prompted us to adopt artificial intelligence model for optimum performance (Osama et al. 2015). ANN models can be trained with different algorithms and can work with noisy data with high nonlinear behaviors. Among the various ANN models, FFBP neural network was utilized in this study and was optimized with the training function 'Train LM'. This supervised training algorithm of Lavenberg-Marquardt is one of the fastest BP algorithms, recommended for optimization which can adjust weights and biases in neural networks (Demuth and Beale 1993). This type of model that utilizes BP algorithm was also found to be effective in predicting Chl content in various growth stages of rice (Liu et al. 2010) and in maize grown under various nitrogen concentrations (Vesali et al. 2015).

Performance of the developed models to predict relative Chl content: The performance evaluation of the developed models suggested improved efficiency of ANN models, compared to linear models to predict the Chl content using RGB features. This was evident with MAE, the most natural and unambiguous approach used to measure the average error magnitude, apart from RMSE (Willmott and Matsuura 2005). However, with the image features of rgb and DGCI-rgb, both types of models showed relatively equal performance as assessed by RMSE and MAE. It appears that the type of image feature may play a contributing role in the performance of the models. The results of this study show that linear regression models developed for the prediction of relative Chl content have a similar performance as ANN models for the image features rgb and DGCI-rgb and are different from the reports of Liu et al. (2010) and Vesali et al. (2015). In their work with field grown rice and maize, they suggested that BP algorithm can predict $\mathrm{Chl}$ content better than a linear model. Noteworthy, image parameters used for the modeling were different from the present study. Liu et al. (2010) used spectral indices in the NIR region and the modeling approach was based on the input luminous factor parameters, Hue, Hue std, $_{\text {, and }} \mathrm{Cr}_{\text {std }}$ similar to Vesali et al. (2015). The present study utilizes the image features based on RGB color space (RGB, rgb) and a combination of HSV and RGB color space model (DGCI-rgb). Such variations in the input data may account for the differences in the model performance. However, as envisaged from Table S3 (supplement), all the models in the present study were capable of predicting the Chl content with PBias $< \pm$ $10 \%$, which indicates the acceptability of the models.

Conclusion: The present study described the modeling approaches using various color indices obtained from digital images captured from smartphone to predict relative $\mathrm{Chl}$ content of rice under field conditions parallel to SPAD measurement. The proposed method of smartphone-based imaging appears to be a cost effective and easy to use alternative to the high cost images captured from digital cameras and satellites. A multiple linear regression model and a FFNN-BP neural network model were developed for the prediction of $\mathrm{Chl}$ content using the selected image derived features. The input variables in the models were RGB, rgb, and DGCI-rgb and the output was predicted relative Chl content which was correlated with the SPADmeasured $\mathrm{Chl}$ content. Both the models were capable of predicting the relative $\mathrm{Chl}$ content. The performance of the model was found to be dependent on the type of image features. ANN models performed better than the linear models with RGB color index, whereas linear regression models were more effective with input of rgb and DGCI-rgb indices. Compared to the conventional sensor-based SPAD meter, implementation of this smartphone-based method as a low cost device can be a potential alternative to estimate relative $\mathrm{Chl}$ content. The developed intelligent image analysis system can be used to measure Chl content within 
the workflow of a typical cultivation system to introduce a decision making step towards plant photosynthetic ability and nutrient status. However, processing of images using in-built processor of the smartphone and the development of an app may make the system amenable for online estimation of relative $\mathrm{Chl}$ content at farmers end.

\section{References}

Agarwal A., Dutta Gupta S.: Assessment of spinach seedling health status and chlorophyll content by multivariate data analysis and multiple linear regression of leaf image features. - Comput. Electron. Agr. 152: 281-289, 2018.

Cabangon R.J., Castillo E.G., Tuong T.P.: Chlorophyll meterbased nitrogen management of rice growing under alternate wetting and drying irrigation. - Field Crop. Res. 121: 136146, 2011.

Cassol D., De Silva F.S.P., Falqueto A.R., Bacarin M.A.: An evaluation of non-destructive methods to estimate total chlorophyll content. - Photosynthetica 46: 634-636, 2008.

Chen P., Haboudane D., Tremblay N. et al.: New spectral indicator assessing the efficiency of crop nitrogen treatment in corn and wheat. - Remote Sens. Environ. 114: 1987-1997, 2010.

Confalonieri R., Foi M., Casa R. et al.: Development of an app for estimating leaf area index using a smartphone. Trueness and precision determination and comparison with other indirect methods. - Comput. Electron. Agr. 96: 67-74, 2013.

Curran P.J., Dungan J.L., Gholz H.L.: Exploring the relationship between reflectance red edge and chlorophyll content in slash pine. - Tree Physiol. 7: 33-48, 1990.

Demuth H., Beale M.: Neural Network Toolbox for Use with MATLAB. The MathWorks, Inc, Natick, USA, 1993.

Dutta Gupta S., Ibaraki Y., Pattanayak A.K.: Development of a digital image analysis method for real time estimation of chlorophyll content in micropropagated potato plants. - Plant Biotechnol. Rep. 7: 91-97, 2013.

Dutta Gupta S., Ibaraki Y., Trivedi P.: Applications of RGB colour imaging in plants. - In: Dutta Gupta S., Ibaraki Y. (ed.): Plant Image Analysis: Fundamentals and Applications. Pp. 41-62. CRC Press, New York 2014.

Dutta Gupta S., Pattanayak A.K.: Intelligent image analysis (IIA) using artificial neural network (ANN) for non-invasive estimation of chlorophyll content in micropropagated plants of potato. - In Vitro Cell. Dev.-Pl. 53: 520-526, 2017.

Errecart M.P., Agnusdei M.G., Lattanzi, F.A., Marino M.A.: Leaf nitrogen concentration and chlorophyll meter readings as predictors of tall fescue nitrogen status. - Field Crop. Res. 129: 46-58, 2012.

Friedrichs A., Busch J.A., van der Woerd H.J., Zielinski, O.: SmartFluo: a method and affordable adapter to measure chlorophyll $a$ fluorescence with smartphones. - Sensors-Basel 17: 678, doi: 10.3390/s17040678, 2017.

Gitelson A.A., Kaufman Y.J., Stark R., Rundquist, D.: Novel algorithms for remote estimation of vegetation fraction. Remote Sens. Environ. 80: 76-87, 2002.

Goetz A.F.H., Rock B.N., Rowan L.C.: Remote sensing for exploration: an overview. - Econ. Geol. 78: 573-590, 1983.

Gómez-Robledo L., López-Ruiz N., Melgosa M. et al.: Using the mobile phone as Munsell soil-colour sensor: an experiment under controlled illumination conditions. - Comput. Electron. Agr. 99: 200-208, 2013.

Gong A., Yu J., He Y., Qiu, Z.: Citrus yield estimation based on images processed by an android mobile phone. - Biosyst. Eng. 115: 162-170, 2013.
Hu H., Zhang J., Sun X., Zhang, X.: Estimation of leaf chlorophyll content of rice using image colour analysis. - Can. J. Remote Sens. 39: 185-190, 2014.

Jinwen, L.: Determination of canopy's average SPAD readings based on the analysis of digital images. - Agrotechnol. 3: 121, doi:10.4172/2168-9881.1000121, 2014.

Karcher D.E., Richardson M.D.: Quantifying turfgrass color using digital image analysis. - Crop Sci. 43: 943-951, 2003.

Kawashima S., Nakatani M.: An algorithm for estimating chlorophyll content in leaves using a video camera. - Ann. Bot.-London 81: 49-54, 1998.

Krogh A.: What are artificial neural networks? - Nat. Biotechnol. 26: 195-197, 2008.

Lee K.J., Lee B.W.: Estimation of rice growth and nitrogen nutrition status using color digital camera image analysis. Eur. J. Agron. 48: 57-65, 2013.

Li F., Mistele B., Hu Y. et al.: Reflectance estimation of canopy nitrogen content in winter wheat using optimized hyperspectral spectral indices and partial least squares regression. - Eur. J. Agron. 52: 198-209, 2014.

Lichtenthaler H.K., Wellburn A.R.: Determinations of total carotenoids and chlorophylls $a$ and $b$ of leaf extracts in different solvents. - Biochem. Soc. T. 11: 591-592, 1983.

Lin F.F., Qiu L.F., Deng J.S. et al.: Investigation of SPAD meterbased indices for estimating rice nitrogen status. - Comput. Electron. Agr. 71: S60-S65, 2010.

Ling Q., Huang W., Jarvis P.: Use of SPAD-502 meter to measure leaf chlorophyll concentration in Arabidopsis thaliana. Photosynth. Res. 107: 209-214, 2011.

Liu Z.A., Yang J.P., Yang Z.C.: Using a chlorophyll meter to estimate tea leaf chlorophyll and nitrogen contents. - J. Soil Sci. Plant Nut. 12: 339-348, 2012.

Liu M., Liu X., Li M. et al.: Neural-network model for estimating leaf chlorophyll concentration in rice under stress from heavy metals using four spectral indices. - Biosyst. Eng. 106: 223$233,2010$.

Markwell J., Octerman J.C., Mitchell J.L.: Calibration of the Minolta SPAD-502 leaf chlorophyll meter. - Photosynth. Res. 46: 467-472, 1995.

Moonrungsee N., Pencharee S., Jakmunee J.: Colorimetric analyzer based on mobile phone camera for determination of available phosphorus in soil. - Talanta 136: 204-209, 2015.

Munoz-Huerta R.F., Guevara-Gonzalez R.G., Contreras-Medina L.M. et al.: A review of methods for sensing the nitrogen status in plants: advantages, disadvantages and recent advances. Sensors 13: 10823-10843, 2013.

Novichonok E.V., Novichonok A.O., Kurbatova J.A., Markovskaya, E.F.: Use of atLEAF+ chlorophyll meter for a nondestructive estimate of chlorophyll content. Photosynthetica 54: 130-137, 2016.

Osama K., Mishra B.N., Somvanshi P.: Machine learning technique in plant biology. - In: Barh, D., Khan M.S., Davies, E. (ed.): Plant Omics: The Omics of Plant Science. Pp. 731754. Springer, India 2015.

Pagola M., Ortiz R., Irigoyen I. et al.: New method to assess barley nitrogen nutrition status based on image colour analysis comparison with SPAD-502. - Comput. Electron. Agr. 65: 213-218, 2009.

Photo Blog Stop. Accessed on August 24, 2017. Retrieved from http://photoblogstop.com/photoshop/accurate-white-balanceadjustments-in-photoshop, 2016.

Pongnumkul S., Chaovalit P., Surasvadi N.: Applications of smartphone-based sensors in agriculture: a systematic review of research. - J. Sensors 15: 18-26, 2015.

Pound M.P., French A.P.: An introduction to images and image analysis. - In: Dutta Gupta S., Ibaraki Y. (ed.): Plant Image 
Analysis: Fundamentals and Applications. Pp. 1-24. CRC Press, New York 2014.

Prasad V.S.S., Dutta Gupta, S.: Applications and potentials of artificial neural networks in plant tissue culture. - In: Dutta Gupta S., Ibaraki Y. (ed.): Plant Tissue Culture Engineering. Pp. 47-67. Springer, Berlin 2008.

RapidTables. Accessed on August 28, 2017. Retrieved from http://www.rapidtables.com/web/color/RGB Color.htm, 2016.

Ravier C., Quemada M., Jeuffroy M.-H.: Use of a chlorophyll meter to assess nitrogen nutrition index during the growth cycle in winter wheat. - Field Crop. Res. 214: 73-82, 2017.

Reyniers M., Walvoort D.J.J., Baardemaaker J.D.: A linear model to predict with a multi-spectral radiometer the amount of nitrogen in winter wheat. - Int. J. Remote Sens. 27: 41594179, 2006.

Riccardi M., Mele G., Pulvento C. et al.: Non-destructive evaluation of chlorophyll content in quinoa and amaranth by simple and multiple regression analysis of RGB image components. - Photosynth. Res. 120: 263-272, 2014.

Rigon J.P.G., Capuani S., Beltrao N.E.M. et al.: Non-destructive determination of photosynthetic pigments in the leaves of castor oil plants. - Acta Sci.-Agron. 34: 325-329, 2012.

Rigon J.P.G., Capuani S., Fernandes D.M., Guimarães, T.M.: A novel method for the estimation of soybean chlorophyll content using a smartphone and image analysis. - Photosynthetica 54: 559-566, 2016.

Rorie R.L., Purcell L.C., Karcher D.E., King, C.A.: The assessment of leaf nitrogen in corn from digital images. Crop Sci. 51: 2174-2180, 2011a.

Rorie R.L., Purcell L.C., Mozaffari M. et al.: Association of 'greenness' in corn with yield and leaf nitrogen concentration. - Agron. J. 103: 529-535, 2011 b.

Santos do Amaral E., Vieira Silva D., Dos Anjos L. et al. : Relationships between reflectance and absorbance chlorophyll indices with RGB (Red, Green, Blue) image components in seedlings of tropical tree species at nursery stage. - New Forest.: doi: 10.1007/s11056-018-9662-4, 2018.

Sim C.C., Zaharah A.R., Tan M.S., Goh, K.J.: Rapid determination of leaf chlorophyll concentration, photosynthetic activity and NK concentration of Elaies guineensis via correlated SPAD502 chlorophyll index. - Asian J. Agr. Res. 9: 132-138, 2015.

Steele M.R., Gitelson A.A., Rundquist D.C.: A comparison of two techniques for nondestructive measurement of chlorophyll content in grapevine leaves. - Agron. J. 100: 779-782, 2008.

Stefan M., Munteanu N., Stoleru V., Mihasan, M.: Effects of inoculation with plant growth promoting rhizobacteria on photosynthesis, antioxidant status and yield of runner bean. Rom. Biotech. Lett. 18: 8132-8143, 2013.

Uddling J., Gelang-Alfredsson J., Piikii K., Pleijel, H.: Evaluating the relationship between leaf chlorophyll concentration and SPAD-502 chlorophyll meter readings. - Photosynth. Res. 91: 37-46, 2007.

Vesali F., Omid M., Kaleita A., Mobli, H.: Development of an android app to estimate chlorophyll content of corn leaves based on contact imaging. - Comput. Electron. Agr. 116: 211220, 2015.

Vesali F., Omid M., Mobli H., Kaleita A.: Feasibility of using smart phones to estimate chlorophyll content in corn plants. Photosynthetica 55: 603-610, 2017.

Vollmann J., Walter H., Sato T., Schweiger, P.: Digital image analysis and chlorophyll metering for phenotyping the effects of nodulation in soybean. - Comput. Electron. Agr. 75: 190195, 2011.

Wang Y., Wang D., Zhang G., Wang, J.: Estimating nitrogen status of rice using the image segmentation of G-R thresholding method. - Field Crop. Res. 149: 33-39, 2013.

Wang Y., Wang D., Shi P., Omasa, K.: Estimating rice chlorophyll content and leaf nitrogen concentration with a digital still colour camera under natural light. - Plant Methods 10: 36, doi: 10.1186/1746-4811-10-36, 2014.

Willmott C.J., Matsuura K.: Advantages of the mean absolute error (MAE) over the root mean square error (RMSE) in assessing average model performance. - Clim. Res. 30: 7982, 2005.

Yadav S.P., Ibaraki Y., Dutta Gupta S.: Estimation of the chlorophyll content of micropropagated potato using RGB based image analysis. - Plant Cell Tiss. Org. 100: 183-188, 2010.

Yuan Z., Ata-Ul-Karim S.T., Cao Q. et al.: Indicators for diagnosing nitrogen status of rice based on chlorophyll meter readings. - Field Crop. Res. 185: 12-20, 2016.

Živković Ž., Mihajlović I., Nikolić D.: Artificial neural network method applied on the nonlinear multivariate problems. Serb. J. Manag. 4: 143-155, 2009.

(C) The authors. This is an open access article distributed under the terms of the Creative Commons BY-NC-ND Licence. 